\title{
ESTUDO DAS PRECIPITAÇÕES MÁXIMAS ANUAIS EM PRESIDENTE PRUDENTE
}

\author{
MARCELO HARTMANN ${ }^{1}$, FERNANDO A. MOALA ${ }^{1}$, MARCO AURÉLIO MENDONÇA $^{2}$ \\ ${ }^{1}$ Universidade Estadual Paulista (UNESP), Faculdade de Ciências e Tecnologia, Departamento de \\ Matemática, Estatística e Computação, Presidente Prudente, SP, Brasil \\ ${ }^{2}$ Universidade Estadual Paulista (UNESP), Faculdade de Ciências e Tecnologia, Departamento de \\ Cartografia, Presidente Prudente, SP, Brasil \\ xmahax@gmail.com, femoala@fct.unesp.br, mammendonca@gmail.com \\ Recebido Outubro 2008 - Aceito Março 2011
}

\begin{abstract}
RESUMO
Considerando a importância sócio-econômica da região de Presidente Prudente, este estudo teve como objetivo estimar a precipitação pluvial máxima esperada para diferentes níveis de probabilidade e verificar o grau de ajuste dos dados ao modelo Gumbel, com as estimativas dos parâmetros obtidas pelo método de máxima verossimilhança. Pelos resultados, o teste de Kolmogorov-Sminorv (K-S) mostrou que a distribuição Gumbel testada se ajustou com p-valor maior que 0.28 para todos os períodos de tempo considerados, comprovando que a distribuição Gumbel apresenta um bom ajustamento aos dados observados para representar as precipitações pluviais máximas. As estimativas de precipitação obtidas pelo método de máxima verossimilhança são consistentes, conseguindo reproduzir com bastante fidelidade o regime de chuvas da região de Presidente Prudente. Assim, o conhecimento da distribuição da precipitação pluvial máxima mensal e das estimativas das precipitações diárias máximas esperadas, possibilita um planejamento estratégico melhor, minimizando assim o risco de ocorrência de perdas econômicas para essa região.

Palavras-Chave: Precipitação máxima, estimador de máxima verossimilhança, distribuição Gumbel, intervalo de confiança.
\end{abstract}

\section{ABSTRACT: STUDY OF THE MAXIMUM ANNUAL PRECIPITATION IN PRESIDENTE PRUDENTE}

Considering the socioeconomic importance of Presidente Prudente area, this study aimed at to estimate the maximum pluvial precipitation expected for different levels of probability and to check the fitting degree of the data to the Gumbel model, with the parameter estimation obtained by the maximum likelihood approach. The Kolmogorov-Sminorv (K-S) test showed that the Gumbel distribution has fitted with p-value larger than 0.28 for all of the time periods considered, showing that the Gumbel distribution presents a good fitting to the observed data representing the maximum pluvial precipitations values. The precipitation estimates obtained by the maximum likelihood approach are consistent allowing to reproduce with plenty reliability the rainfall regime for Presidente Prudente region. Therefore, the knowledge of the monthly maximum pluvial precipitation distribution and the expected maximum daily precipitation estimates permits a better strategic planning thus minimizing the risk of economical losses for this region.

Keywords: Maximum precipitation, maximum likelihood estimate, Gumbel distribution, confidence interval. 


\section{INTRODUÇÃO}

A caracterização da precipitação pluvial constitui um importante elemento de apoio para realização de obras hidráulicas, planejamento e gestão de recursos hídricos, e nas atividades da defesa civil e público em geral. Dentre as características de grande interesse das precipitações está o estudo da distribuição da precipitação pluvial máxima. Com esse conhecimento pode-se evitar problemas como: erosão do solo, inundações em áreas rurais e urbanas, queda agrícola, danificações de estradas, prejuízos em projetos de obras hidráulicas, danos em sistemas de drenagem, dentre outros (Vieira et al., 1991).

O acúmulo das águas pluviais em épocas de chuva intensa, em Presidente Prudente e região, têm prejudicado sensivelmente a economia local, devido a enchentes causadas na área urbana e perda de colheita na zona rural. Portanto, o estudo da precipitação pluvial máxima na região de Presidente Prudente-SP é de grande importância na elaboração de projetos agrícolas e de obras para controle de enchentes.

Sendo a ocorrência de precipitação pluvial máxima um processo aleatório, que não permite uma previsão determinística com grande antecedência, o ajustamento de um modelo probabilístico que melhor descreva o processo se faz necessário, a fim de se fazer previsões.

Vários trabalhos na literatura têm mostrado que a distribuição de probabilidade Gumbel é a que melhor se ajusta aos fenômenos meteorológicos, entre os quais a precipitação pluvial máxima. Beijo et al. (2003) estimaram a precipitação pluvial diária máxima esperada em diferentes níveis de probabilidade para a região de Jaboticabal (SP), ajustando a distribuição de Gumbel. As estimativas dos parâmetros são obtidas pelo método dos momentos e de máxima verossimilhança com os dados de precipitação pluvial diária máxima, coletados de jan/1956 a dez/2001, e agrupados em períodos mensal e anual. Ferreira et al. (2005) confirmaram esse bom ajuste da distribuição Gumbel para dados de precipitação máxima de 165 localidades do Estado de São Paulo. Mello e Silva (2005) também ajustaram a distribuição Gumbel à série histórica de precipitação máxima diária anual da região Alto Rio Grande. Eles estudaram os efeitos que os métodos de estimação, Máxima Verossimilhança e Momentos, dos parâmetros da distribuição de Gumbel proporcionam na estimativa da equação de chuvas intensas, aplicando-se um modelo chuva-vazão. Mais recentemente, Sansigolo (2008), ao comparar o ajustamento de diversas distribuições de probabilidade como Normal, Gumbel, Fréchet, Weibull, Log-Normal e Pearson, aos dados de precipitação máxima diária e de temperaturas máximas absolutas, coletados na cidade de Piracicaba, SP (1917-2006), mostrou que a distribuição Gumbel teve o melhor ajustamento.
Assim, tendo em vista a importância do conhecimento das possíveis precipitações mensais máximas, este trabalho tem como objetivos estimar a precipitação pluvial diária máxima esperada para diferentes períodos e níveis de probabilidade, para a região de Presidente Prudente, e verificar o nível de ajuste dos dados ao modelo Gumbel. Para isto, é utilizado o método de máxima verossimilhança para estimação dos parâmetros do modelo e o teste de Kolmogorov-Smirnov, para verificar o ajustamento em todos os períodos de chuva considerados.

\section{MATERIAL E MÉTODO}

\subsection{Base de dados}

Os dados de precipitação pluviométrica utilizados na pesquisa foram obtidos da Estação Meteorológica da Faculdade de Ciências e Tecnologia da Universidade Estadual Paulista, campus de Presidente Prudente (SP), compreendendo um período de 57 anos, de janeiro de 1947 a dezembro de 2003. O conjunto de dados de precipitação pluvial máxima, representando uma amostra, é composto do maior valor diário registrado no mês para cada ano.

\subsection{A distribuição Gumbel}

Adistribuição Gumbel tem apresentado grande importância no estudo de eventos extremos ocorridos na natureza, e tem sido aplicada com grande freqüência na análise da precipitação pluvial máxima. Nesse trabalho, os testes Kolmogorov-Smirnov e QuiQuadrado são utilizados para checar se a distribuição Gumbel é realmente apropriada para valores de precipitação máxima observados em Presidente Prudente, concordando assim os resultados encontrados na literatura.

A distribuição Gumbel, também conhecida como distribuição valor extremo, surge como distribuição limite para máximos ou mínimos (valores extremos) de uma amostra de variáveis aletórias independentes, identicamente distribuídas, quando o tamanho da amostra aumenta. A distribuição Gumbel para valores máximos tem a função densidade de probabilidade dada por:

$$
f(x \mid \phi, \varphi)=\frac{1}{\varphi} \exp \left\{-\frac{x-\phi}{\varphi}-\exp \left\{-\frac{x-\phi}{\varphi}\right\}\right\},
$$

para $-\infty<x<+\infty,-\infty<\phi<+\infty$ e $\varphi>0$, onde $\phi$ é o parâmetro de posição e $\varphi$ é o parâmetro de escala.

A probabilidade $P$ de que ocorra uma precipitação pluvial máxima maior que um certo valor $x$ é dada por,

$$
P=P\{X>x\}=1-\exp \left\{-\exp \left\{-\frac{x-\phi}{\varphi}\right\}\right\},
$$

em que $x$ é a precipitação máxima do período e $0<x<+\infty$. 
O nível de retorno associado ao período de retorno $t=1 / P$ é dado por

$$
x_{p}=\phi-\varphi \log (-\log (1-P))
$$

\subsection{Análise estatística dos dados}

A análise estatística dos dados foi realizada primeiramente verificando o nível de ajuste dos dados ao modelo Gumbel através dos testes Kolmogorov-Smirnov e Qui-quadrado a um nível de significância de 5\%, para verificar o ajustamento em todos os períodos de chuva considerados. Para isto, foi utilizado o método de máxima verossimilhança para estimação dos parâmetros do modelo. Após o ajustamento da distribuição Gumbel aos dados, o objetivo foi então, estimar pontualmente e por intervalo, a precipitação pluvial diária máxima esperada para diferentes períodos e níveis de probabilidade.

\subsubsection{Estimação pontual}

Considerando uma amostra aleatória $x_{1}, x_{2}, \ldots, x_{n}$ obtida da distribuição Gumbel em (Equação 1), a função de verossimilhança para os parâmetros $\phi$ e $\varphi$ é simplesmente o produto das densidades de cada uma das observações na amostra, dada então por,

$$
L(\phi, \varphi \mid x)=\frac{1}{\varphi^{n}} \exp \left\{-\frac{n}{\varphi}(\bar{x}-\phi)-\sum_{i=1}^{n} \exp \left\{-\frac{x_{i}-\phi}{\varphi}\right\}\right\},
$$

O estimador de máxima-verossimilhança é obtido como segue. Da Equação 4, a função log-verossimilhança é dada por

$$
\ell(\phi, \varphi \mid x)=\log L(\phi, \varphi \mid x)=-n \log \varphi-\frac{n}{\varphi}(\bar{x}-\phi)-\sum_{i=1}^{n} \exp \left\{-\frac{x_{i}-\phi}{\varphi}\right\} .
$$

Os estimadores de máxima verossimilhança $\hat{\phi}$ e $\hat{\varphi}$ são obtidos resolvendo as equações de verossimilhança $\partial \ell / \partial \phi=0$ e $\partial \ell / \partial \varphi=0$ simultaneamente. Segue então que:

$$
e^{\hat{\phi}}=\left[\frac{1}{n} \sum_{i=1}^{n} \exp \left\{-\frac{x_{i}}{\hat{\varphi}}\right\}\right]^{-\hat{\phi}}
$$

Substituindo a Equação 6 em $\partial \ell / \partial \varphi=0$, obtém-se:

$$
\hat{\varphi}+\frac{\sum_{i=1}^{n} x_{i} \exp \left\{-\frac{x_{i}}{\hat{\varphi}}\right\}}{\sum_{i=1}^{n} \exp \left\{-\frac{x_{i}}{\hat{\varphi}}\right\}}-\bar{x}=0 .
$$

Uma vez que as Equações 6 e 7 não podem ser resolvidas analiticamente para $\hat{\phi}$ e $\hat{\varphi}$, um método numérico deve ser utilizado, por exemplo, Método de Newton-Raphson (Ruggiero e Lopes, 1988). Portanto, primeiramente determina-se $\hat{\varphi}$ como solução da
Equação 7 e então, substitui-se este valor na Equação 6 para obter $\hat{\phi}$.

Da Equação 2, o estimador de máxima verossimilhança da probabilidade $P$ é dado por

$$
\hat{P}=1-\exp \left\{-\exp \left\{-\frac{x-\hat{\phi}}{\hat{\varphi}}\right\}\right\} \text {. }
$$

A precipitação pluvial máxima esperada pode ser estimada também usando a função inversa da Equação 2, fixando-se os níveis de probabilidade $P$. Assim, a precipitação pluvial máxima provável para uma determinada probabilidade pode ser determinada pela seguinte expressão:

$$
\hat{x}_{p}=\hat{\phi}-\hat{\varphi} \log (-\log (1-P)) \text {. }
$$

\subsubsection{Estimação por intervalos}

A estimação pontual não permite julgar qual a possível magnitude do erro cometido, isto é, não dá uma idéia acerca da maior ou menor diferença entre a estimativa do parâmetro de interesse e seu verdadeiro valor. Então, a inferência pode ser complementada com a construção de intervalos de confiança, de forma que valores dentro do intervalo seriam melhores estimadores dos parâmetros do que valores fora do intervalo.

A estimação com intervalos de confiança para os parâmetros do modelo pode ser obtida pela aproximação normal assintótica dos estimadores de máxima verossimilhança, isto é,

$$
(\hat{\phi}, \hat{\varphi}) \sim N_{2}\left\lfloor(\phi, \varphi), I^{-1}(\hat{\phi}, \hat{\varphi})\right\rfloor,
$$

para $n \rightarrow \infty$, onde $I^{-1}(\hat{\phi}, \hat{\varphi})$ é a matriz de Informação de Fisher dada por

$$
I(\phi, \varphi)=\left[\begin{array}{cc}
\frac{n}{\varphi^{2}} & -\frac{n}{\varphi^{2}}[1+\psi(1)] \\
-\frac{n}{\varphi^{2}}[1+\psi(1)] & \frac{n}{\varphi^{2}}\left[1+\psi(2)+\psi^{2}(2)\right]
\end{array}\right],
$$

e $\psi(k)$ é a função digama $\psi(k)=\frac{d}{d k} \Gamma(k)$, conforme definido em Lawless (1982).

Assim, as regiões de confiança conjunta aproximadas para $\phi$ e $\varphi$ podem ser obtidas. Intervalos de confiança individuais aproximados para os parâmetros $\phi$ e $\varphi$ e com coeficiente de confiança $100(1-\alpha) \%$ são dados por

$$
\begin{gathered}
\hat{\phi}-z_{\frac{\alpha}{2}} \sqrt{\operatorname{var}(\hat{\phi})}<\phi<\hat{\phi}+z_{\frac{\alpha}{2}} \sqrt{\operatorname{var}(\hat{\phi})} \\
\hat{\varphi}-z_{\frac{\alpha}{2}} \sqrt{\operatorname{var}(\hat{\varphi})}<\varphi<\hat{\varphi}+z_{\frac{\alpha}{2}} \sqrt{\operatorname{var}(\hat{\varphi})} \\
\text { onde }\left[\begin{array}{cc}
\operatorname{var}(\hat{\phi}) & \operatorname{cov}(\hat{\phi}, \hat{\varphi}) \\
\operatorname{cov}(\hat{\phi}, \hat{\varphi}) & \operatorname{var}(\hat{\varphi})
\end{array}\right]=I^{-1}(\hat{\phi}, \hat{\varphi})=\frac{\hat{\varphi}}{n}\left[\begin{array}{cc}
1.109 & 0,257 \\
0,257 & 0,608
\end{array}\right], \text { para “var” e }
\end{gathered}
$$


"cov" indicando, respectivamente, a variância e a covariância entre os estimadores.

Para se obter o intervalo de confiança para o p-ésimo quantil $x_{p}=\phi-\varphi w_{p}$, onde $w_{p}=\log (-\log (1-P))$, trata-se $\left(\hat{x}_{p}-x_{p}\right) / \sqrt{\operatorname{var}\left(\hat{x}_{p}\right)}$ como uma distribuição normal padrão, $\mathrm{N}(0$, $1)$, onde $\operatorname{var}\left(\hat{x}_{p}\right)=\operatorname{var}(\hat{\phi})+w_{p}^{2} \operatorname{var}(\hat{\varphi})-2 w_{p} \operatorname{cov}(\hat{\phi}, \hat{\varphi})$. Assim, o intervalo de confiança $100(1-\alpha) \%$ para $x_{p}$ é dado por:

$$
\hat{x}_{p}-z_{\frac{\alpha}{2}} \sqrt{\operatorname{var}\left(\hat{x}_{p}\right)}<x_{p}<\hat{x}_{p}-z_{\frac{\alpha}{2}} \sqrt{\operatorname{var}\left(\hat{x}_{p}\right)}
$$

$\operatorname{com} \hat{x}_{p}=\hat{\phi}-\hat{\varphi} w_{p}, \hat{\phi}$ e $\hat{\varphi}$ como soluções das Equações 6 e 7.

\section{RESULTADOS E DISCUSSÃO}

Os dados de precipitação pluvial diária foram agrupados em períodos mensais, extraindo-se a máxima precipitação pluvial diária observada de cada mês.

Uma das pressuposições para que se possa ajustar modelos probabilísticos, como a distribuição Gumbel, é que as observações sejam aleatórias. Assim, inicialmente foi realizado um run-test para verificar a aleatoriedade das precipitações máximas observadas para cada um dos doze meses e também para a série de máximas anuais. Os resultados do teste revelam que as treze séries consideradas apresentam um comportamento aleatório, como mostrado na Tabela 1 .

A Figura 1 representa um gráfico temporal dos valores de precipitações máximas anuais relativas aos anos estudados. Observa-se que não há uma tendência crescente ou decrescente na precipitação máxima, ou seja, um aumento ou decréscimo nos valores de chuvas máximas mensais na região de Presidente Prudente.

Ainda pela Figura 1, nota-se que durante o período de 57 anos do estudo poucas vezes ocorreram chuvas com índices acima de $100 \mathrm{~mm}$, indicando que estes ocorreram devido a causas aleatórias. Para analisar a presença ou não de possíveis tendências nas séries de máxima mensal, foi utilizado o Teste de Tendência de Mann-Kendall. A Tabela 1 fornece os p-valores para cada mês dos respectivos testes.

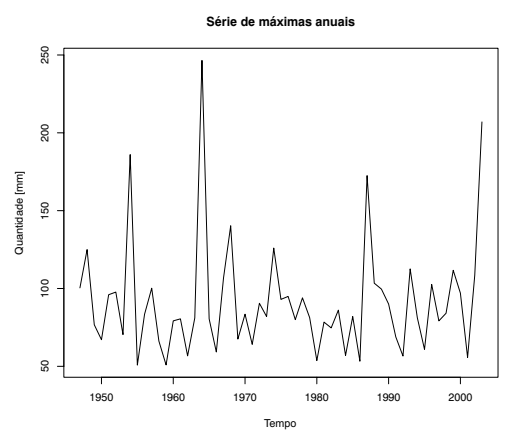

Figura 1: Representação gráfica dos dados de chuva diária máxima mensal de 1947 a dezembro de 2003 em Presidente Prudente-SP.
Os resultados indicam que não houve tendência significativa na precipitação máxima observada em nenhuma das treze séries.

A Figura 2 representa uma comparação gráfica temporal dos valores máximos de precipitações máximas mensais relativas aos anos estudados, considerando-se os meses de cada estação do ano. Pode-se observar também, uma nítida variação quantitativa de chuvas no decorrer do ano, cujos meses mais chuvosos são, sistematicamente, janeiro e fevereiro, com máximas atingindo até $250 \mathrm{~mm}$. Há uma estação menos chuvosa no inverno, com precipitações máximas mensais ao redor de 40 mm. Neste caso, Agosto e Setembro são os meses com menos precipitação.

A distribuição Gumbel tem sido aplicada com grande freqüência na análise estatística da precipitação pluvial máxima. Para Presidente Prudente e região, a distribuição Gumbel também apresentou melhor ajustamento aos dados observados para representar as precipitações pluviais máximas. $\mathrm{O}$ teste de Kolmogorov-Sminorv mostrou que a distribuição Gumbel testada se ajustou com p-valor maior que 0.28 para todos os períodos de tempo considerados, ou seja, com boa aderência, contudo seu pior ajustamento ocorreu para os dados do mês de Julho, onde teve o menor p-valor, resultado esse idêntico ao obtido por Gomes, et al. (1989).

A Tabela 2 apresenta os estimadores de máximaverossimilhança para os parâmetros da distribuição Gumbel com seus respectivos intervalos de confiança e os p-valores para os testes de aderência de Kolmogorov-Smirnov (K-S) e Qui-quadrado $\left(\chi^{2}\right)$, para cada mês do ano.

Na Tabela 2, observa-se que as estimativas dos parâmetros $\phi$ e $\varphi$ foram menores nos períodos mais secos (outono e inverno), e maiores nos períodos mais chuvosos. Obviamente, isto se deve a ocorrência de precipitação máxima menores nos meses secos.

Tabela 1 - P-valor dos testes de aleatorização e teste de tendência para os dados pluviométricos mensais de Presidente Prudente e região-SP.

\begin{tabular}{|l|c|c|}
\hline \multicolumn{1}{|c|}{ Meses } & Run Teste & $\begin{array}{c}\text { Teste de Mann- } \\
\text { Kendall }\end{array}$ \\
\hline Janeiro & 0,34 & 0,45 \\
\hline Fevereiro & 0,59 & 0,16 \\
\hline Março & 0,68 & 0,75 \\
\hline Abril & 0,21 & 0,27 \\
\hline Maio & 0,42 & 0,22 \\
\hline Junho & 0,79 & 0,19 \\
\hline Julho & 0,22 & 0,51 \\
\hline Agosto & 0,27 & 0,55 \\
\hline Setembro & 0,98 & 0,19 \\
\hline Outubro & 0,22 & 0,08 \\
\hline Novembro & 0,89 & 0,95 \\
\hline Dezembro & 0,41 & 0,40 \\
\hline Anual & $\mathbf{0 . 9 0}$ & $\mathbf{0 . 5 6}$ \\
\hline
\end{tabular}



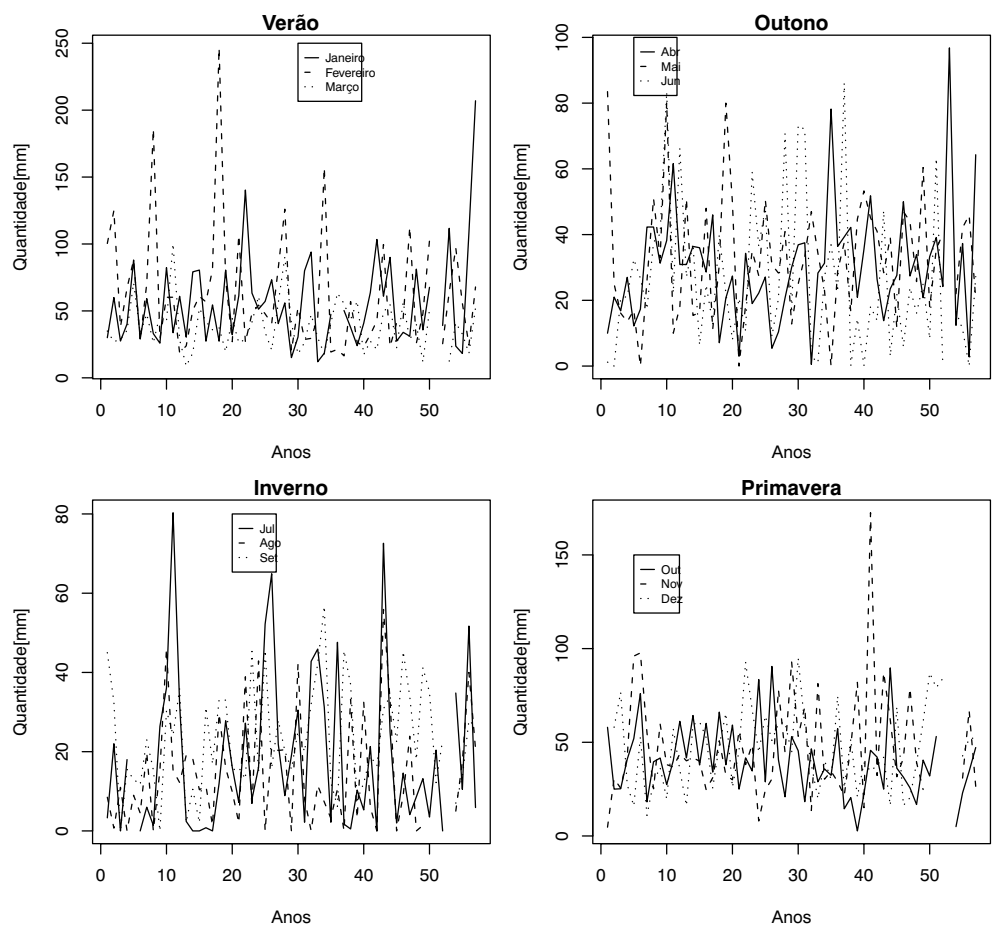

Figura 2 - Comparação gráfica da precipitação pluviométrica mensal entre 1947 e 2003 em Presidente Prudente-SP.

Tabela 2 - P-valor dos testes de ajustamento K-S e $\chi^{2}$, estimadores de máxima verossimilhança e intervalos de confiança (IC) $95 \%$ para os parâmetros $\phi$ e $\varphi$ da distribuição Gumbel ajustada para os dados pluviométricos de Presidente Prudente e região-SP.

\begin{tabular}{|l|c|c|c|c|c|c|}
\hline \multicolumn{1}{|c|}{ Meses } & K-S & $\chi^{2}$ & $\hat{\varphi}$ & IC 95\% & $\hat{\phi}$ & IC 95\% \\
\hline Janeiro & 0,459 & 0,141 & 23,314 & $(18,99 ; 27,63)$ & 41,452 & $(35,98 ; 46,92)$ \\
\hline Fevereiro & 0,287 & 0,057 & 27,303 & $(22,28 ; 32,32)$ & 43,374 & $(37,02 ; 49,72)$ \\
\hline Março & 0,913 & 0,670 & 14,342 & $(11,68 ; 17,00)$ & 30,241 & $(26,87 ; 33,60)$ \\
\hline Abril & 0,607 & 0,386 & 14,115 & $(11,73 ; 16,95)$ & 22,461 & $(19,15 ; 25,76)$ \\
\hline Maio & 0,904 & 0,808 & 15,798 & $(12,89 ; 18,70)$ & 23,952 & $(20,28 ; 27,62)$ \\
\hline Junho & 0,662 & 0,788 & 16,058 & $(13,10 ; 19,01)$ & 16,467 & $(12,73 ; 20,20)$ \\
\hline Julho & 0,327 & 0,239 & 12,970 & $(10,56 ; 15,37)$ & 10,101 & $(7,06 ; 13,14)$ \\
\hline Agosto & 0,686 & 0,208 & 10,241 & $(8,32 ; 12,15)$ & 8,662 & $(6,24 ; 11,09)$ \\
\hline Setembro & 0,768 & 0,145 & 11,554 & $(9,43 ; 13,67)$ & 16,955 & $(14,27 ; 19,64)$ \\
\hline Outubro & 0,954 & 0,206 & 16,127 & $(13,14 ; 19,12)$ & 31,085 & $(27,30 ; 34,87)$ \\
\hline Novembro & 0,745 & 0,167 & 19,446 & $(15,77 ; 23,12)$ & 34,581 & $(29,93 ; 39,23)$ \\
\hline Dezembro & 0,704 & 0,360 & 18,291 & $(14,87 ; 21,71)$ & 36,694 & $(32,36 ; 41,02)$ \\
\hline
\end{tabular}

Um dos métodos gráficos mais utilizados na verificação do ajustamento de uma dada distribuição aos dados observados é o Quantil-Quantil Plot ou Q-Q plot. O procedimento empregado consiste na comparação gráfica dos quantis teóricos da distribuição Gumbel com os quantis dos dados amostrais, mostrando a linearidade entre os dados ajustados e os empíricos, de forma que quantos mais próximos os pontos da linha de referência maior é a certeza de que os dados ajustados se comportam em relação à determinada distribuição.

A Figura 3 representa um Q-Q plot para a distribuição Gumbel ajustada. O gráfico mostra que a maioria dos pontos do Q-Q plot estão sensivelmente ao longo de uma reta, havendo apenas uma relação não-linear bastante acentuada entre os quantis teóricos e empíricos nas caudas das distribuições, o que implica que o ajustamento da distribuição Gumbel à série estudada é perfeitamente recomendável. Os meses que 

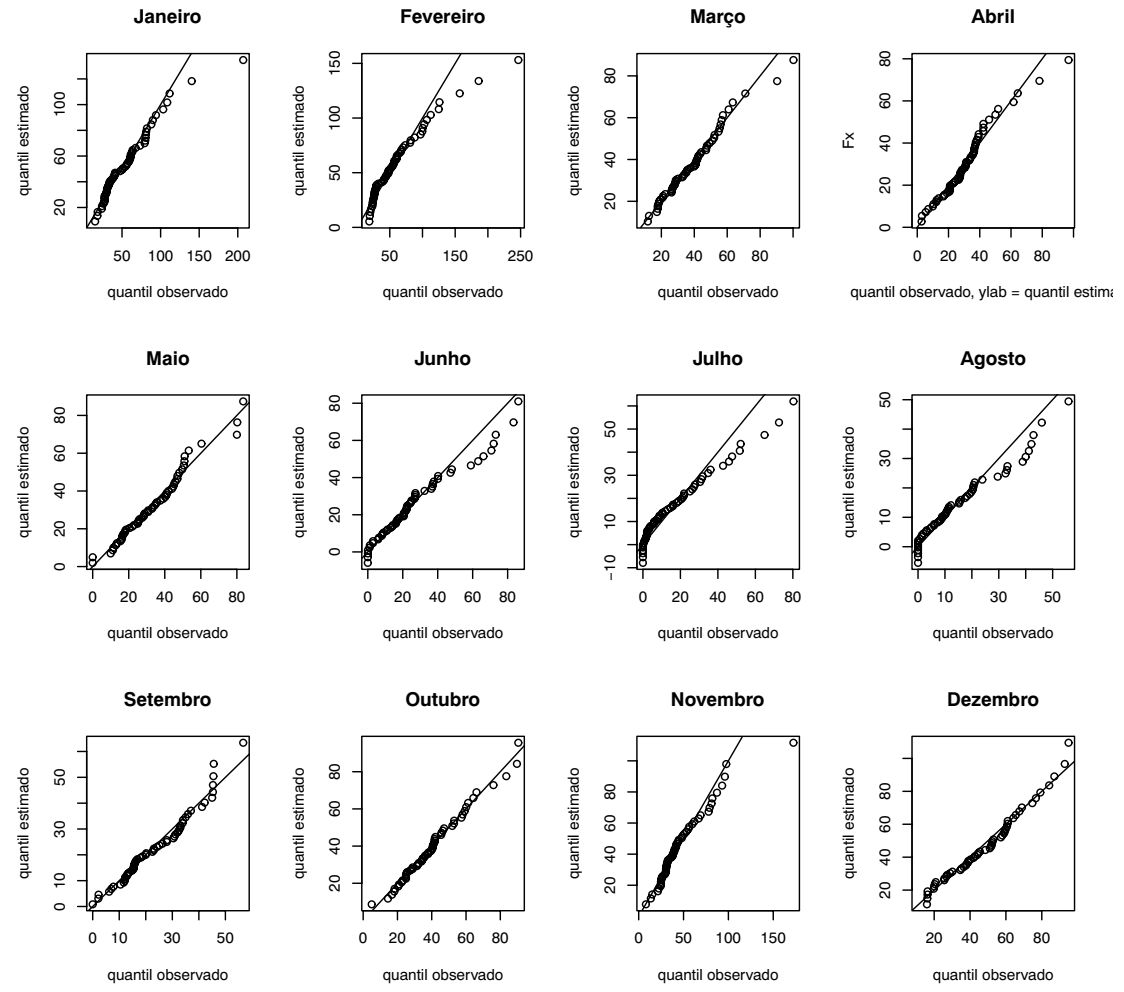

Figura 3 - "Quantil-Quantil Plot” para a distribuição Gumbel ajustadada.
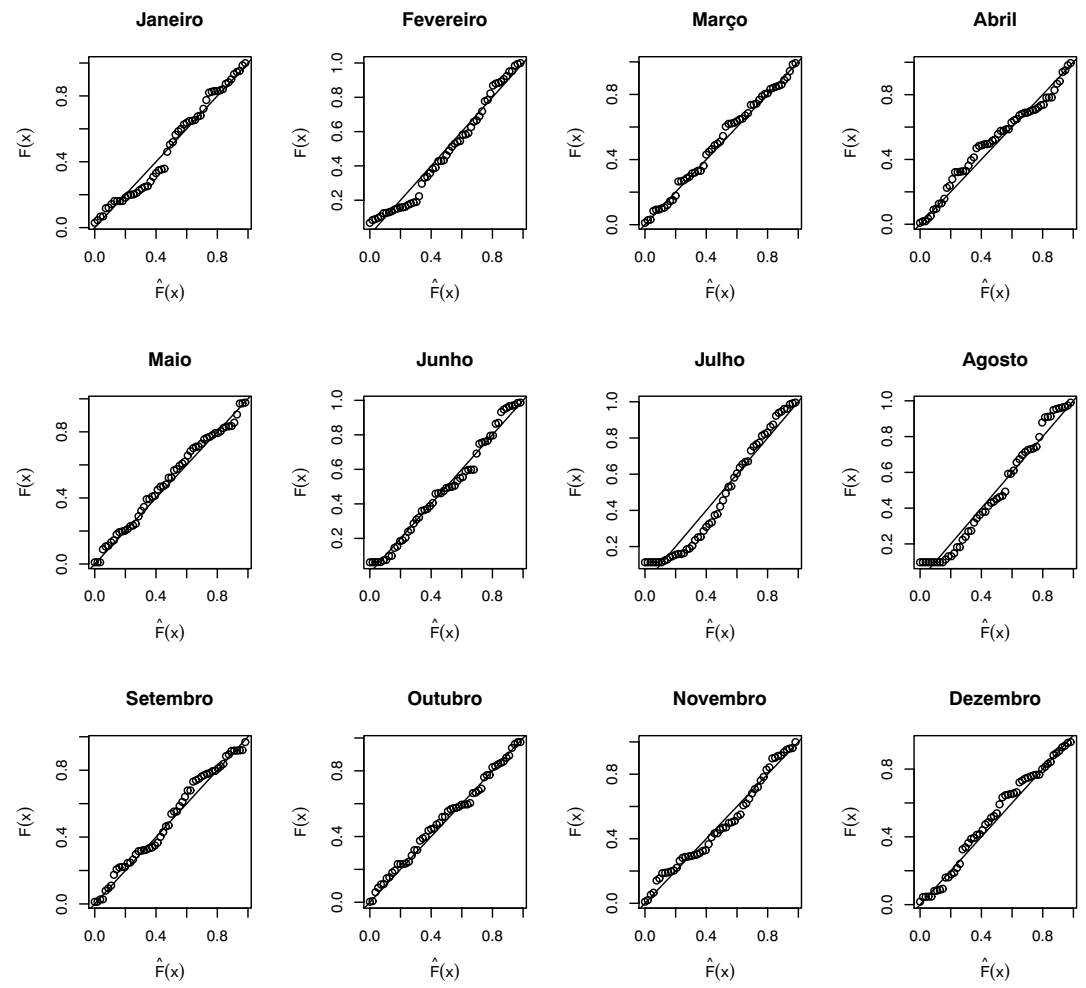

Figura 4 - Gráficos das distribuições acumuladas empírica e teórica ajustadas. 
Tabela 3 - Estimativas das precipitações diárias máximas esperadas, nos períodos de um mês e um ano em 6 níveis de probabilidade, calculadas pela distribuição Gumbel, usando-se os estimadores $\hat{\phi}$ e $\hat{\varphi}$ de máxima verossimilhança em Presidente Prudente-SP.

\begin{tabular}{|l|l|l|l|l|l|l|c|c|}
\hline Estações & \multicolumn{7}{|c|}{ Nível de Probabilidade } & \multicolumn{3}{|c|}{ IC 95\% } \\
\hline & $\mathbf{5 0 \%}$ & $\mathbf{7 5 \%}$ & $\mathbf{9 0 \%}$ & $\mathbf{9 5 \%}$ & $\mathbf{9 7 , 5 \%}$ & $\mathbf{9 9 \%}$ & $x_{0.95}$ & $x_{0.99}$ \\
\hline Verão & $\mathbf{4 9 , 2 7}$ & $\mathbf{4 2 , 6 9}$ & $\mathbf{3 7 , 8 6}$ & $\mathbf{3 5 , 3 6}$ & $\mathbf{3 3 , 3 9}$ & $\mathbf{3 1 , 2 8}$ & $\mathbf{( 2 7 , 0 7 ; 4 3 , 6 5 )}$ & $\mathbf{( 2 1 , 4 4 ; 4 1 , 1 1 )}$ \\
\hline Janeiro & 53,61 & 46,59 & 41,45 & 38,79 & 36,68 & 34,43 & $(31,84 ; 45,74)$ & $(26,18 ; 42,68)$ \\
\hline Fevereiro & 57,61 & 49,39 & 43,37 & 40,25 & 37,78 & 35,15 & $(32,11 ; 48,39)$ & $(25,49 ; 44,81)$ \\
\hline Março & 37,72 & 33,40 & 30,24 & 28,60 & 27,30 & 25,92 & $(24,32 ; 32,88)$ & $(20,84 ; 30,99)$ \\
\hline Outono & $\mathbf{2 8 , 9 7}$ & $\mathbf{2 4 , 2 3}$ & $\mathbf{2 0 , 7 6}$ & $\mathbf{1 8 , 9 6}$ & $\mathbf{1 7 , 5 4}$ & $\mathbf{1 6 , 0 2}$ & $\mathbf{( 1 4 , 7 2 ; 2 3 , 1 1 )}$ & $\mathbf{( 1 1 , 0 4 ; 2 0 , 9 9 )}$ \\
\hline Abril & 29,82 & 25,57 & 22,46 & 20,85 & 19,57 & 18,21 & $(16,64 ; 25,06)$ & $(13,22 ; 23,20)$ \\
\hline Maio & 32,19 & 27,43 & 23,95 & 22,15 & 20,72 & 19,19 & $(17,44 ; 26,86)$ & $(13,60 ; 24,78)$ \\
\hline Junho & 24,84 & 20,00 & 16,46 & 14,63 & 13,18 & 11,63 & $(9,84 ; 19,42)$ & $(5,95 ; 17,31)$ \\
\hline Inverno & $\mathbf{1 7 , 9 9}$ & $\mathbf{1 4 , 3 3}$ & $\mathbf{1 1 , 6 5}$ & $\mathbf{1 0 , 2 6}$ & $\mathbf{9 , 1 7}$ & $\mathbf{7 , 9 9}$ & $\mathbf{( 6 , 5 5 ; 1 3 , 9 7 )}$ & $\mathbf{( 3 , 5 9 ; 1 2 , 3 9 )}$ \\
\hline Julho & 16,86 & 12,96 & 10,10 & 8,62 & 7,44 & 6,19 & $(4,75 ; 12,49)$ & $(1,60 ; 10,78)$ \\
\hline Agosto & 14,00 & 10,92 & 8,66 & 7,49 & 6,56 & 5,58 & $(4,43 ; 10,54)$ & $(1,96 ; 9,20)$ \\
\hline Setembro & 22,98 & 19,50 & 16,95 & 15,63 & 14,59 & 13,47 & $(12,18 ; 19,07)$ & $(9,38 ; 17,56)$ \\
\hline Primavera & $\mathbf{4 3 , 3 8}$ & $\mathbf{3 7 , 9 7}$ & $\mathbf{3 4 , 0 0}$ & $\mathbf{3 1 , 9 5}$ & $\mathbf{3 0 , 3 2}$ & $\mathbf{2 8 , 5 9}$ & $\mathbf{( 2 5 , 3 5 ; 3 8 , 5 5 )}$ & $(\mathbf{2 0 , 7 6 ; 3 6 , 4 2 )}$ \\
\hline Outubro & 39,49 & 34,64 & 31,08 & 29,24 & 27,78 & 26,23 & $(24,43 ; 34,05)$ & $(20,52 ; 31,93)$ \\
\hline Novembro & 44,72 & 38,86 & 34,58 & 32,36 & 30,60 & 28,73 & $(26,56 ; 38,16)$ & $(21,85 ; 35,61)$ \\
\hline Dezembro & 46,23 & 40,72 & 36,69 & 34,60 & 32,95 & 31,19 & $(29,14 ; 40,05)$ & $(24,72 ; 37,66)$ \\
\hline
\end{tabular}

mostraram um menor alinhamento dos valores ajustados correspondem aos meses menos chuvosos Junho, Julho e Agosto.

Outra forma de avaliar graficamente o ajuste da distribuição é a utilização dos gráficos de probabilidades (PP plot). Neste caso, o ajuste dos dados pode ser comparado em termos da probabilidade acumulada, comparando a função de distribuição acumulada (FDA) empírica e a função de distribuição acumulada ajustada pela distribuição Gumbel. Através da Figura 4, observam-se os pontos alinhados em uma reta indicando um bom ajuste do modelo, isto é, dos dados à distribuição valor extremo.

Utilizando-se a distribuição Gumbel com as estimativas dos parâmetros obtidos pelo método da máxima verossimilhança, dadas na Tabela 2, calculou-se a precipitação pluvial diária máxima provável para períodos de retorno de 2, 4, 10, 20, 40 e 50 anos ou, respectivamente níveis de probabilidade: 50, 75, $90,95,97.5$ e 98 por cento nos períodos mensais. Os intervalos de confiança $95 \%$ associados aos períodos de retorno $\chi_{0,95}$ e $\chi_{0,99}$ anos também são avaliados, revelando que os maiores níveis de retorno registram-se nos meses de Janeiro e Fevereiro. Os resultados são apresentados na Tabela 3 .

As informações contidas na Tabela 3 podem ser interpretadas como, as estimativas das precipitações diárias máximas no período de um mês e um ano. Por exemplo, considerando o mês de Janeiro e o nível de probabilidade de
$95 \%$, verifica-se que a precipitação máxima esperada é maior que $38 \mathrm{~mm}$, ou seja, espera-se que em 1 de cada 20 anos, o valor da precipitação diária máxima em Janeiro seja superior a $38 \mathrm{~mm}$.

\section{CONCLUSÕES}

Os resultados mostram que houve um bom ajuste da distribuição Gumbel para os dados da precipitação máxima mensal para a região de Presidente Prudente. As estimativas de precipitação obtidas pelo método de máxima verossimilhança são consistentes conseguindo reproduzir com bastante fidelidade o regime de chuvas da região de Presidente Prudente.

O conhecimento da distribuição da precipitação pluvial máxima mensal, e consequentemente as estimativas das precipitações diárias máximas esperadas possibilitam planejar a época adequada de plantio e colheita além de auxiliar no planejamento de obras de engenharia hidráulica e agrícola na região de Presidente Prudente, minimizando assim o risco de ocorrência de perdas econômicas.

\section{REFERÊNCIAS}

BEIJO, L.A.; MUNIZ, J.A.; VOLPE, C.A.; PEREIRA, G.T. Estudo da precipitação máxima em Jaboticabal (SP) pela distribuição de Gumbel utilizando dois métodos de estimação 
dos parâmetros. Revista Brasileira de Agrometeorologia, Santa Maria, v. 11, n. 1, p. 141-147, 2003.

FERREIRA, J.C.; DANIEL, L.A.; TOMAZELA, M. Parâmetros para equações mensais de estimativas de precipitação de intensidade máxima para o Estado de São Paulo - Fase I. Ciência e Agrotecnologia. v. 29, n. 6, 2005.

GOMES, F.G.; AQUINO, L.H.; OLIVEIRA, M.S. Estudo da distribuição e freqüência de precipitação pluviométrica máxima em períodos de dez e quinze dias, um mês e um ano, em Lavras (MG) pela distribuição Gumbel. Ciência e Prática, Lavras, v. 13, n. 2, p. 177-184, 1989.

LAWLESS, J.F. Statistical Models and Methods for Lifetime Data. Wiley, New York. 1982.

MELLO, C.R.; SILVA, A.M. Métodos estimadores dos parâmetros da distribuição de Gumbel e sua influência em estudos hidrológicos de projeto. Irriga, Botucatu, v. 10, n. 4, p. 318-334, 2005.
RUGGIERO, M A.G.; LOPES, V.L.R. Cálculo numérico: aspectos teóricos e computacionais. São Paulo: McGrawHill, 1988.

SANSIGOLO, C.A. Distribuições de extremos de precipitação diária, temperatura máxima e mínima e velocidade do vento em Piracicaba, SP (1917-2006). Revista Brasileira de Meteorologia, v. 23, n. 3, p. 341-346, 2008.

VIEIRA, S.R.; LOMBARDI NETO, F.; BURROWS, I.T. Mapeamento da chuva diária máxima provável para o Estado de São Paulo. Revista Brasileira de Ciência do Solo, Campinas, v. 15, p. 93-98, 1991. 